\title{
Hadronic Spectrum of a Holographic Dual of QCD
}

\author{
Guy F. de Téramond ${ }^{1}$ and Stanley J. Brodsky ${ }^{2}$ \\ ${ }^{1}$ Universidad de Costa Rica, San José, Costa Rica \\ ${ }^{2}$ Stanford Linear Accelerator Center, Stanford University, Stanford, California 94309, USA
}

\begin{abstract}
We compute the spectrum of light hadrons in a holographic dual of QCD defined on $A d S_{5} \times S^{5}$ which has conformal behavior at short distances and confinement at large interquark separation. Specific hadrons are identified by the correspondence of string modes with the dimension of the interpolating operator of the hadron's valence Fock state. Higher orbital excitations are matched quanta to quanta with fluctuations about the AdS background. Since only one parameter, the QCD scale $\Lambda_{\mathrm{QCD}}$, is used, the agreement with the pattern of physical states is remarkable. In particular, the ratio of delta to nucleon trajectories is determined by the ratio of zeros of Bessel functions.
\end{abstract}

The correspondence [1] between 10-dimensional string theory defined on anti-de Sitter $\left(A d S_{5} \times S^{5}\right)$ and YangMills theories at its conformal $3+1$ space-time boundary 2] has led to important insights into the properties of QCD at strong coupling. As shown by Polchinski and Strassler [3], one can give a nonperturbative derivation of dimensional counting rules [4] for the leading powerlaw fall-off of hard exclusive glueball scattering in gauge theories with a mass gap dual to supergravity in warped space-times. The resulting theories have the hard behavior expected from QCD at short distances, rather than the soft behavior characteristic of string theory. Another important application is the description of deep inelastic scattering structure functions at small $x[\underline{5}$ ]. One can also derive the falloff of hadronic light-front wavefunctions in QCD at large transverse momentum by matching their short-distance properties to the behavior of the string solutions in the large- $r$ conformal region of AdS space [6]. The scale dependence of the string modes determines the analytic behavior of the QCD hadronic wave function, providing a precise counting rule for each Fock component with any number of quarks and gluons and any internal orbital angular momentum [6]. The predicted orbital dependence coincides with perturbative QCD results [7].

The $\mathcal{N}=4$ super Yang-Mills (SYM) theory at large $N_{C}$ in four dimensions is dual to the low energy supergravity approximation to type IIB string compactified on $A d S_{5} \times S^{5}$ [1]. However, QCD is fundamentally different from SYM theories where all of the matter fields appear in adjoint multiplets of $S U\left(N_{C}\right)$. The introduction of quarks in the fundamental representation is dual to the introduction of an open string sector [8].

There is now substantial theoretical [9] and empirical 10] evidence that the QCD coupling has an IR fixed point. In many phenomenological applications, such as exclusive processes at experimentally accessible momentum transfers, the amplitudes are evaluated in the regime where the exchanged gluon momenta are not very large and the QCD coupling is nearly constant and not small [1]. For example, the phenomenological successes of dimensional counting rules for exclusive processes can be understood if QCD resembles a strongly coupled confor- mal theory at moderate but not asymptotic momentum transfer. QCD is also a confining gauge theory in the infrared with a mass gap $\Lambda_{\mathrm{QCD}}$ and a well-defined spectrum of color-singlet hadronic states.

The isomorphism of the group $S O(2,4)$ of conformal QCD in the limit of massless quarks and vanishing $\beta$ function with the isometries of AdS space, $x^{\mu} \rightarrow \lambda x^{\mu}$, $r \rightarrow r / \lambda$, maps scale transformations into the holographic coordinate $r$ : the string mode in $r$ is the extension of the hadron wave function into the fifth dimension. Different values of $r$ correspond to different energy scales at which the hadron is examined, and determines the scale of the invariant separation between quarks $x_{\mu} x^{\mu} \rightarrow \lambda^{2} x_{\mu} x^{\mu}$. In particular, the $r \rightarrow \infty$ boundary corresponds to the $Q \rightarrow$ $\infty$, zero separation limit. Conversely, color confinement implies that there is a maximum separation of quarks and a minimum value of $r$. Thus, AdS space should end at a finite value $r_{0}=\Lambda_{\mathrm{QCD}} R^{2}$ truncating the regime where the string modes can propagate. The cutoff at $r_{0}$ breaks conformal invariance and allows the introduction of the QCD scale.

A 10-dimensional nonconformal metric dual to a confining gauge theory is written as $\underline{3}$ ]

$$
d s^{2}=\frac{R^{2}}{z^{2}} e^{2 A(z)}\left(\eta_{\mu \nu} d x^{\mu} d x^{\nu}-d z^{2}\right)+d s_{X}^{2},
$$

where $A(z) \rightarrow 0$ as $z=R^{2} / r \rightarrow 0$, and $R$ is the AdS radius. The metric (1) behaves asymptotically as a product of AdS space and a compact manifold $X$. Color confinement will be described in a simplified model based on a "hard-wall" approximation where the metric factor $e^{2 A(z)}$ is a step function. This provides an analog of the MIT bag model where quarks are permanently confined inside a finite region of space [12]. As in the bag model the linearized equations in the bulk have no interactions. However, unlike bag models, the truncated boundary conditions on string modes are imposed on the holographic coordinate, not on the bag wave function at fixed time. The truncated anti-de Sitter/conformal field theory (CFT) thus provides a manifestly Lorentz invariant model with confinement at large distances and conformal behavior at short distances. 
The AdS/CFT correspondence can be interpreted in the present context as a classical duality between the valence state of a hadron in the asymptotic $3+1$ boundary and the lightest mass string mode in $A d S_{5} \times S^{5}$ [6, 13]. Higher Fock components are manifestations of the quantum fluctuations of QCD; metric fluctuations of the bulk geometry about the fixed AdS background should correspond to quantum fluctuations of Fock states above the valence state. In fact, as shown by Gubser, Klebanov and Polyakov for large Lorentz spin, orbital excitations in the boundary correspond to string degrees of freedom propagating in the bulk from quantum fluctuations in the AdS sector 14]. We identify the higher spin hadrons with the fluctuations around the spin $0, \frac{1}{2}, 1$ and $\frac{3}{2}$ string solutions on $A d S_{5}$. This identification avoids the huge string dimensions associated with spin $>2$, which grow as $\Delta \sim\left(g_{s} N_{C}\right)^{\frac{1}{4}}$ at large $N_{C}$. The interpolating operators $\mathcal{O},\langle P|\mathcal{O}| 0\rangle \neq 0$, which couple to the color-singlet hadrons at the boundary can be constructed from gaugeinvariant products of local quark and gluon fields taken at the same point in four-dimensional space-time. We introduce quarks in the fundamental representation at the AdS boundary, and follow their wavefunctions as they propagate into the bulk.

As a first application of our procedure, consider the twist (dimension minus spin) two glueball interpolating operators $\mathcal{O}_{4+L}=F D_{\left\{\ell_{1}\right.} \ldots D_{\left.\ell_{m}\right\}} F$, written in terms of the symmetrized product of covariant derivatives $D$. The operator $\mathcal{O}_{4+L}$ has total internal space-time orbital momentum, $L=\sum_{i=1}^{m} \ell_{i}$ and conformal dimension $\Delta=4+L$. We shall match the large $r$ asymptotic behavior of each string mode in the bulk to the corresponding conformal dimension of the boundary operators of each hadronic state while maintaining conformal invariance [13]. In the conformal limit, an $L$ quantum, which is identified with a quantum fluctuation about the AdS geometry, corresponds to an effective five-dimensional mass $\mu$ in the bulk side. The allowed values of $\mu$ are uniquely determined by requiring that asymptotically the dimensions become spaced by integers, according to the spectral relation $(\mu R)^{2}=\Delta(\Delta-4)$. For large spacetime angular momentum $L$, we recover the string theory results for the spectrum of oscillatory exited states $\mu \simeq L / R$. The physical string modes are plane waves along the Poincaré coordinates with four-momentum $P_{\mu}$ and hadronic invariant mass states given by $P_{\mu} P^{\mu}=\mathcal{M}^{2}$. The four-dimensional mass spectrum $\mathcal{M}_{L}$ follows from the boundary condition $\Phi\left(x, z_{o}\right)=0$ on the solutions of the AdS wave equation with effective mass $\mu$ :

$$
\left[z^{2} \partial_{z}^{2}-(d-1) z \partial_{z}+z^{2} \mathcal{M}^{2}-(\mu R)^{2}\right] f(z)=0,
$$

where $\Phi(x, z)=e^{-i P \cdot x} f(z)$. The normalizable modes are

$$
\Phi_{\alpha, k}(x, z)=C_{\alpha, k} e^{-i P \cdot x} z^{2} J_{\alpha}\left(z \beta_{\alpha, k} \Lambda_{Q C D}\right),
$$

with $C_{\alpha, k}$ a normalization constant, $\alpha=2+L$ and
$\Delta=4+L$ for $d=4$. For small $z$, $\Phi$ scales as $z^{-\Delta}$, where the scaling dimension $\Delta$ of the string mode has the same dimension of the interpolating operator which creates a hadron. The four-dimensional mass spectrum is then determined by the zeros of Bessel functions $\beta_{\alpha, k}$ :

$$
\mathcal{M}_{\alpha, k}=\beta_{\alpha, k} \Lambda_{\mathrm{QCD}}
$$

A similar expression for the glueball spectrum follows from considering a AdS slice with boundary conditions at some finite value of $z$ [15].

We next consider the twist-two, dimension $3+L$, vector-meson operators $\mathcal{O}_{3+L}^{\mu}=\bar{\psi} \gamma^{\mu} D_{\left\{\ell_{1}\right.} \ldots D_{\left.\ell_{m}\right\}} \psi$, dual to string modes $\Phi_{\mu}=e^{-i P \cdot x} f_{\mu}(z)$ propagating on AdS space with polarization along the Poincaré coordinates. The string wavefunctions of the vector mesons are then determined by the five-dimensional wave equation

$$
\left[z^{2} \partial_{z}^{2}-(d-1) z \partial_{z}+z^{2} \mathcal{M}^{2}-(\mu R)^{2}+d-1\right] f_{\mu}(z)=0
$$

in the $\Phi_{z}=0$ gauge [16], with normalizable modes

$$
\Phi_{\alpha, k}^{\mu}(x, z)=C_{\alpha, k} e^{-i P \cdot x} z^{2} J_{\alpha}\left(z \beta_{\alpha, k} \Lambda_{Q C D}\right) \epsilon^{\mu},
$$

where $\alpha=1+L$ and $\Delta=3+L$. The hadronic mass spectrum follows from $\Phi_{\mu}\left(x, z_{o}\right)=0$. Similarly, the pseudoscalar mesons are described by the operator $\mathcal{O}_{3+L}=\bar{\psi} \gamma_{5} D_{\left\{\ell_{1}\right.} \ldots D_{\left.\ell_{m}\right\}} \psi$, dual to string modes polarized along the radial coordinate in the $\Phi_{\mu}=0$ gauge. The predicted spectrum is compared in Fig. 11 with the masses of light mesons listed by the Particle Data Group [17. We plot the values of $\mathcal{M}^{2}$ as function of $L$ for $\Lambda_{\mathrm{QCD}}=0.263 \mathrm{GeV}$. The predicted masses for the lightest hadrons are too high, but otherwise the results are in good agreement with the empirical values. A string mode with a node in the coordinate $r$ should correspond to a radial resonance with a node in the interquark separation. The first radial AdS eigenvalue has a mass 1.8 $\mathrm{GeV}$ which is high compared to the masses of the observed radial excited mesons, the $\pi(1300)$ or the $\rho(1450)$. These defects could possibly be cured by modifying the sharp cutoff at $r_{0}$.

The study of the baryon spectrum is crucial for our understanding of bound states of strongly interacting relativistic confined particles. Consider the twistthree, dimension $\frac{9}{2}+L$, baryon operators $\mathcal{O}_{(9 / 2)+L}=$ $\psi D_{\left\{\ell_{1}\right.} \ldots D_{\ell_{q}} \psi D_{\ell_{q+1}} \ldots D_{\left.\ell_{m}\right\}} \psi$, dual to spin- $\frac{1}{2}$ or $\frac{3}{2}$ modes in the bulk. In this case, we need to solve the full ten-dimensional Dirac wave equation, $\not D \hat{\Psi}=0$, since the lowest Kaluza-Klein (KK) mode of the Dirac operator on an N-sphere is not zero. Consequently, baryons are charged under the $S U(4)_{R} \sim S O(6) R$ symmetry of $S^{5}$. In contrast, the $S U(4)_{R}$ charge of mesons is zero. We have classified the baryonic states according to the $S U(2)_{F} \otimes S U(2)_{\text {spin }} \subset S U(4)$ isospin-spin symmetry corresponding to two massless quarks. 


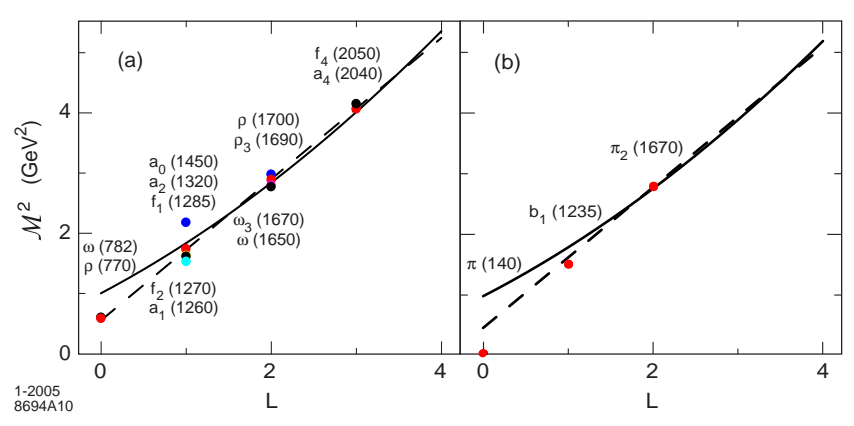

FIG. 1: Light meson orbital states for $\Lambda_{\mathrm{QCD}}=0.263 \mathrm{GeV}$. Results for the vector mesons are shown in (a) and for the pseudoscalar mesons in (b). The dashed line corresponds to the usual linear Regge trajectory and has slope $1.16 \mathrm{GeV}^{2}$.
The field $\hat{\Psi}$ can be expanded in terms of eigenfunctions $\eta_{\kappa}(y)$ of the Dirac operator on the compact space $X$, $i D_{X} \eta_{k}(y)=\lambda_{\kappa} \eta_{\kappa}(y)$, with eigenvalues $\lambda_{\kappa}$ as $\hat{\Psi}(x, z, y)=$ $\sum_{\kappa} \Psi_{\kappa}(x, z) \eta_{\kappa}(y)$, where the $y$ are coordinates of $X$. The AdS Dirac equation is 16

$$
\left[z^{2} \partial_{z}^{2}-d z \partial_{z}+z^{2} \mathcal{M}^{2}-\left(\lambda_{\kappa}+\mu\right)^{2} R^{2} y+\frac{d}{2}\left(\frac{d}{2}+1\right)+\left(\lambda_{\kappa}+\mu\right) R \hat{\Gamma}\right] f(z)=0
$$

where $\Psi(x, z)=e^{-i P \cdot x} f(z)$ and $\hat{\Gamma} u_{ \pm}= \pm u_{ \pm}$. For $A d S_{5}$, $\hat{\Gamma}$ is the four-dimensional chirality operator $\gamma_{5}$. The AdS mass $\mu$ is determined asymptotically by the orbital excitations in the boundary: $\mu=L / R$. The eigenvalues on $S^{d+1}$ are $\lambda_{\kappa} R= \pm\left(\kappa+\frac{d}{2}+\frac{1}{2}\right), \kappa=0,1,2, \ldots$ [18]. The normalizable modes for $\kappa=0$ are

$$
\begin{array}{r}
\Psi_{\alpha, k}(x, z)=C_{\alpha, k} e^{-i P \cdot x} z^{5 / 2}\left[J_{\alpha}\left(z \beta_{\alpha, k} \Lambda_{\mathrm{QCD}}\right) u_{+}(P)\right. \\
\left.+J_{\alpha+1}\left(z \beta_{\alpha, k} \Lambda_{\mathrm{QCD}}\right) u_{-}(P)\right]
\end{array}
$$

where $u^{-}=\frac{\gamma^{\mu} P_{\mu}}{P} u^{+}, \alpha=2+L$ and $\Delta=\frac{9}{2}+L$. The solution of the spin- $\frac{3}{2}$ Rarita-Schwinger equation in AdS space is more involved, but considerable simplification occurs in the $\Psi_{z}=0$ gauge for polarization along Minkowski coordinates, $\Psi_{\mu}$, where it becomes similar to the spin- $\frac{1}{2}$ solution [19]. The four-dimensional spectrum follows from $\Psi^{ \pm}\left(x, z_{o}\right)=0$ or $\Psi_{\mu}^{ \pm}\left(z, z_{o}\right)=0$

$$
\mathcal{M}_{\alpha, k}^{+}=\beta_{\alpha, k} \Lambda_{\mathrm{QCD}}, \quad \mathcal{M}_{\alpha, k}^{-}=\beta_{\alpha+1, k} \Lambda_{\mathrm{QCD}},
$$

with a scale independent mass ratio. Two of the fermions can be assigned to the fundamental representation of $S U\left(N_{C}\right)$; however, to have a color-singlet state of three fields at large $N_{C}$, the third must be in the $N_{C}\left(N_{C}-1\right) / 2$ antisymmetric representation [20]. For $N_{C}=3$ we recover the usual interpolating operator which creates a physical baryon in $Q C D(3+1): \mathcal{O}_{9 / 2}=\epsilon_{a b c} \psi_{a} \psi_{b} \psi_{c}$. The internal spin $S$ of a given hadron matches the spin of its dual string. The boundary conditions are $\Psi^{+}\left(x, z_{o}\right)=0$ for baryons with internal spin $S=\frac{1}{2}$ and $\Psi_{\mu}^{-}\left(x, z_{o}\right)=0$ for $S=\frac{3}{2}$. Figure 2(a) shows the predicted orbital spectrum of the nucleon states and Fig. [2(b) the $\Delta$ orbital resonances. The only parameter is the value of $\Lambda_{\mathrm{QCD}}$ which we take as $0.22 \mathrm{GeV}$. The baryon states with internal spin $S=\frac{1}{2}$ lie on a curve below the states with
$S=\frac{3}{2}$. The spectrum shows a clustering of states with the same orbital $L$, consistent with a strongly suppressed spin-orbit force. Nucleon and $\Delta$ resonances with same total quark spin $S$ fall on the same trajectory. With the exception of the lowest states, the agreement of the predicted spectrum with data is remarkable. However, the first AdS radial state has a mass $1.85 \mathrm{GeV}$, so it is difficult to identify it with the Roper $N \frac{1}{2}^{+}(1440)$.

Eq. (9) predicts a novel parity degeneracy between states in the parallel trajectories shown in Fig. 2] as seen by displacing the upper curve by one unit of $L$ to the right. Thus, the $L=1$ states $N(1650), N(1675)$ and $N(1700)$ are degenerate with the $L=2, N(1680)$ and $N(1720)$, likewise the $L=3$ states $N(2190)$ and $N(2250)$ with the $L=4, N(2220)$. The deltas provide another excellent example of parity degeneracy: the $L=2$ states $\Delta(1905), \Delta(1910), \Delta(1920), \Delta(1950)$ are within the error bars degenerate with the $L=3$ state $\Delta(1930)$. It has been suggested that parity doublets with the same total angular momentum, such as the $\Delta \frac{5}{2}^{+}(1905)-\Delta \frac{5}{2}^{-}(1930)$ doublet, are due to chiral symmetry restoration in the upper part of the light baryon spectrum [21], which is consistent with the larger symmetry implied by (9). In the quark-diquark model of Jaffe and Wilczek [22], baryon states on the lower trajectory of Fig. 22 (a), correspond to "good" diquarks, the upper to "bad" diquarks, and all the states shown in Fig. 2(b) to "bad" diquarks, with exception of the $\Delta(1930)$ which does not follow the simple $3 q$ quark-diquark pattern.

The general agreement of the holographic model with the known light baryon spectrum is quite remarkable and nontrivial. The only mass scale in the holographic model is $\Lambda_{\mathrm{QCD}}$. The best fit to the meson spectra is $\Lambda_{\mathrm{QCD}}=$ $263 \mathrm{MeV}$; the best fit to the baryon spectrum is $\Lambda_{\mathrm{QCD}}=$ 


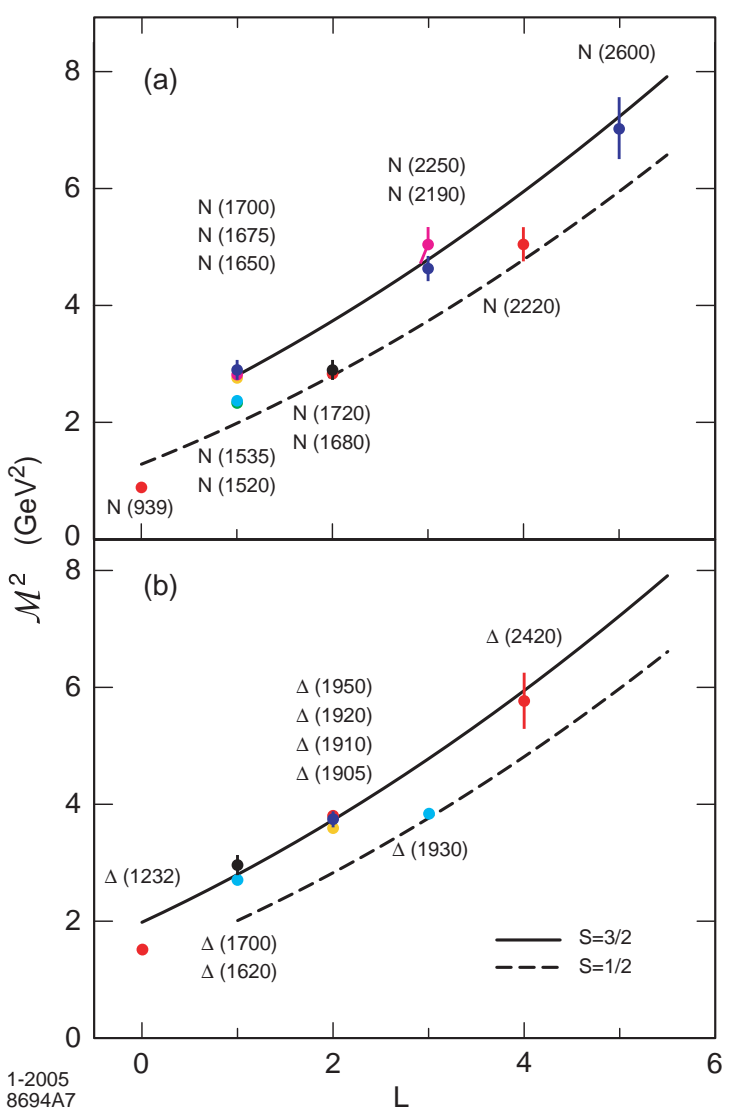

FIG. 2: Light baryon orbital spectrum for $\Lambda_{\mathrm{QCD}}=0.22 \mathrm{GeV}$. Predictions for the nucleons are shown in (a) and for the $\Delta$ trajectories in (b). The lower dashed curves correspond to baryon states dual to spin- $\frac{1}{2}$ modes in the bulk and the upper continuous curve to states dual to spin- $\frac{3}{2}$ modes.

$220 \mathrm{MeV}$. The small difference could be due to the different sensitivity of the mesons and baryons to the space truncations at $z_{0}=1 / \Lambda_{\mathrm{QCD}}$. Moreover, the ratio of the delta to nucleon trajectories is parameter independent, depending simply on the ratios of zeros of Bessel functions. Hadrons are identified by requiring that the state in the bulk has the correct matching conformal dimension at $z \rightarrow 0, x^{2} \rightarrow 0$. Our baryon analysis is thus based on color-singlet states which extrapolate to three fermion fields at zero separation. The contributions of higher particle Fock states of a hadron wave function are suppressed by extra powers of $z$ at $z \rightarrow 0$, so only the valence state is important in the short-distance domain.

The holographic model is relevant to the color-singlet hadronic spectrum of any gauge theory which has conformal scaling at short distances and confinement at large distances. The degeneracy of the hadronic states depends on the flavor symmetry that is assumed; i.e., the number of massless quarks. There is no explicit dependence on $N_{C}$, and the QCD spectrum follows by matching dimensions to $S U(3)_{C}$ color-singlet hadronic states at the $z \rightarrow 0$ boundary. For example, the 10-dimensional low- est Dirac AdS modes have dimension 9/2, precisely the conformal dimension of a $S U(3)_{C} 3$-quark baryon state.

The SYM particles are expected to acquire a mass of the order of the supersymmetric (SUSY) breaking scale and decouple from the theory. Consequently, the only (non-supersymmetric) hadronic states which can be derived from the classical holographic theory are effectively the (dimension-3) $J^{P}=0^{-}, 1^{-}$pseudoscalar and vector mesons, the (dimension- $\frac{9}{2}$ ) $J^{P}=\frac{1}{2}^{+}, \frac{3}{2}^{+}$baryons, and the (dimension-4) $J^{P}=0^{+}$glueball states - corresponding exactly to the lowest-mass physical hadronic states. Our prediction for the mass of the lowest glueball state $\Theta^{++}$is $\mathcal{M} \simeq 1.3 \mathrm{GeV}$ for $\Lambda_{\mathrm{QCD}}=0.26 \mathrm{GeV}$. Hadrons with nonzero orbital angular momentum and higher Fock states require the introduction of quantum fluctuations. The model provides an explanation of why hadrons consist of two gluons, three quarks, or a quark and antiquark, and not other exotic combinations.

In some sense the holographic model is a covariant generalization of the MIT/SLAC bag models; however, unlike the bag models, it also incorporates the nearconformal behavior of QCD at short distances. The approach is highly successful in organizing the hadron spectrum, although it underestimates the spin-orbit separations of the $L=1$ orbital states. The model might be improved for the low-lying states by modifying the boundary conditions at $r=r_{0}$. Our results suggest that basic features of the QCD hadron spectrum can be understood in terms of a higher dimensional dual theory.

We thank James D. Bjorken, Elena Caceres, Simon Capstick, Lance Dixon, Leonid Glozman, Joe Polchinski, David Richards and Matt Strassler for helpful comments. This research was supported in part by the National Science Foundation under Grant No. PHY9907949 and by the Department of Energy contract DEAC02-76SF00515.

[1] J. M. Maldacena, Adv. Theor. Math. Phys. 2, 231 (1998).

[2] S. S. Gubser, I. R. Klebanov and A. M. Polyakov, Phys. Lett. B 428, 105 (1998); E. Witten, Adv. Theor. Math. Phys. 2, 253 (1998).

[3] J. Polchinski and M. J. Strassler, Phys. Rev. Lett. 88, 031601 (2002).

[4] S. J. Brodsky and G. R. Farrar, Phys. Rev. Lett. 31, 1153 (1973); V. A. Matveev, R. M. Muradian and A. N. Tavkhelidze, Lett. Nuovo Cimento 7, 719 (1973).

[5] J. Polchinski and M. J. Strassler, JHEP 0305, 012 (2003).

[6] S. J. Brodsky and G. F. de Téramond, Phys. Lett. B 582, 211 (2004).

[7] X. d. Ji, J. P. Ma and F. Yuan, Phys. Rev. Lett. 90, 241601 (2003).

[8] D. J. Gross and H. Ooguri, Phys. Rev. D 58, 106002 (1998); E. Witten, JHEP 9807, 006 (1998).

[9] See, e.g., R. Alkofer, C. S. Fischer and F. J. Llanes- 
Estrada, Phys. Lett. B 611, 279 (2005).

[10] S. J. Brodsky, hep-ph/0408069

[11] S. J. Brodsky, C. R. Ji, A. Pang and D. G. Robertson, Phys. Rev. D 57, 245 (1998).

[12] A. Chodos, R. L. Jaffe, K. Johnson, C. B. Thorn and V. F. Weisskopf, Phys. Rev. D 9, 3471 (1974).

[13] G. F. de Téramond and S. J. Brodsky, hep-th/0409074

[14] S. S. Gubser, I. R. Klebanov and A. M. Polyakov, Nucl. Phys. B 636, 99 (2002).

[15] H. Boschi-Filho and N. R. F. Braga, Eur. Phys. J. C 32, 529 (2004); JHEP 0305, 009 (2003).
[16] W. Muck and K. S. Viswanathan, Phys. Rev. D 58, 106006 (1998).

[17] S. Eidelman et al. [Particle Data Group Collaboration], Phys. Lett. B 592, 1 (2004).

[18] R. Camporesi and A. Higuchi, gr-qc/9505009

[19] A. Volovich, JHEP 9809, 022 (1998).

[20] S. Dimopoulos, S. Raby and L. Susskind, Nucl. Phys. B 173, 208 (1980).

[21] L. Y. Glozman, Phys. Lett. B 475, 329 (2000).

[22] F. Wilczek, hep-ph/0409168 